\title{
Présence de Mesocyclops ogunnus Onabamiro, 1957 (Crustacea : Copepoda) en Tunisie
}

\author{
S. Mouelhi ${ }^{1}$ \\ D. Defaye ${ }^{2}$ \\ G. Balvay ${ }^{3}$
}

Mots-clés : Copepoda, Cyclopoida, Mesocyclops ogunnus, biogéographie, Tunisie.

Le cyclopoïde Mesocyclops ogunnus Onabamiro, 1957 a été identifié dans la retenue de Sidi Salem (Tunisie). C'est la première observation d'une espèce du genre Mesocyclops en Tunisie. Déjà signalé en Algérie, cette nouvelle observation permet de confirmer le lien entre les aires de répartition africaine intertropicale et euro-asiatique de M. ogunnus (Israël, Ouzbékistan, Bangladesh, Sri Lanka, Laos).

\section{Occurrence of Mesocyclops ogunnus Onabamiro, 1957 (Crustacea : Copepoda) in Tunisia}

Keywords : Copepoda, Cyclopoida, Mesocyclops ogunnus, biogeography, Tunisia.

Mesocyclops ogunnus (Cyclopoida) is reported from the Sidi Salem reservoir (Tunisia). This is the first record of Mesocyclops from Tunisia and the previous discovery of M. ogunnus in Algeria confirms the link between its distribution areas from the African continent (inter-tropical zone) to the Euro-asiatic zone (Israel, Uzbekistan, Bangladesh, Sri Lanka, Laos).

\section{Introduction}

Le genre Mesocyclops Sars, 1914 est représenté en Afrique par douze espèces, redécrites ou décrites par Van de Velde (1984). Au cours de sa révision, cet auteur établit, sur la base de la redéfinition des caractères spécifiques et après Kiefer (1981), que l'espèce Mesocyclops leuckarti (Claus, 1857), considérée jusqu'alors

1. Faculté des Sciences de Tunis, Département de Biologie, 2092 El Manar II, Tunisie. E-mail : f.charfi@ fst.rnu.tn

2. Muséum National d'Histoire Naturelle, Laboratoire de ZoologieArthropodes, 61 rue de Buffon, F-75231 Paris Cedex 05. E-mail : ddefaye@cimrsl.mnhn.fr

3. INRA Station d'Hydrobiologie Lacustre, B.P. 511, F-74203

Thonon-les-Bains Cedex. E-mail : balvay@thonon.inra.fr comme une espèce cosmopolite, est absente en Afrique, son aire de répartition étant limitée à l'Europe et à la partie occidentale de l'Asie du Nord. Selon cet auteur, les sites africains renfermant le genre Mesocyclops sont localisés essentiellement dans la zone intertropicale, ce genre étant inconnu dans le Maghreb comme dans la péninsule ibérique, le sud de la Grèce et de l'Italie ainsi que dans les îles méditerranéennes. En conséquence, les identifications de $M$. leuckarti en Afrique doivent être considérées comme douteuses et leur statut spécifique confirmé (Dussart \& Defaye 1985). Ainsi, Roy \& Gauthier (1927) citent M. leuckarti dans l'oued Nil (nord algérien), espèce non confirmée ultérieurement par Gauthier (1928) et qui pour Van de Velde (1984) résulterait d'une erreur d'identification.

Samraoui et al. (1998) découvrent Mesocyclops ogunnus en Algérie au lac Oubeira, au lac Bleu, ainsi 
qu'à Garaet El Khobzi et au lac Ochrea (zones humides de El Kala). M. salinus ssp. (?) est également identifié au lac Tonga. Sohlobji et al. (1993) signalent à leur tour la présence de $M$. leuckarti dans la retenue de $\mathrm{Si}$ di Salem (Tunisie septentrionale) à partir de matériel récolté en 1989-1990. Enfin, dans leur inventaire bibliographique du zooplancton des eaux continentales de Tunisie, Mouelhi et al. (1999) signalent la découverte d'une espèce (non précisée) appartenant au genre Mesocyclops.

La vérification du matériel récolté entre 1995 et 1998 dans la retenue du barrage de Sidi Salem et des collections de Sohlobji (1992) établit la présence de Mesocyclops ogunnus dans le domaine pélagique de ce réservoir dès 1989.

\section{Biogéographie}

Récoltée pour la première fois en 1951 au Nigeria (Onabamiro 1952, 1957), cette espèce est largement répartie en Afrique comme Van de Velde (1984) l'a montré (Bénin, Egypte, Côte d'Ivoire, Sénégal, HauteVolta, Kenya, Mali, Mauritanie, Mozambique, Soudan, Tchad, Afrique du Sud, Ethiopie et Kenya). Des observations ultérieures ont permis de confirmer la présence de cette espèce en Ethiopie (Defaye 1988), au Niger (Jeje 1988), en Côte d'Ivoire (Dumont \& Maas 1988, Bonou et al. 1991, Pagano \& Saint-Jean 1994) et au Sénégal (Defaye 1991).

Sur le continent euro-asiatique, cette espèce a été identifiée en Israël (Por 1984, Gophen 1988), au Bangladesh (Dussart \& Fernando 1988), en Ouzbékistan (Mirabdullayev 1996), au Laos (Reid \& Kay 1992) et au Sri Lanka (Dussart \& Fernando 1985). Reid \& Pinto-Coelho (1994) mentionnent également $M$. ogunnus au Brésil, mais émettent l'hypothèse d'une possible introduction de cette espèce sur le continent sud-américain.

\section{Description du lieu de récolte}

La retenue de Sidi Salem est située à $60 \mathrm{~km}$ environ au sud-ouest de Tunis, près de l'agglomération de Testour (Gouvernorat de Béja) ; elle a une superficie de 4208 ha, une capacité maximale de $561.10^{6} \mathrm{~m}^{3}$ et une profondeur moyenne d'environ $13 \mathrm{~m}$ (coordonnées : $36^{\circ} 35^{\prime} 20^{\prime \prime} \mathrm{N}, 9^{\circ} 23^{\prime} 45^{\prime \prime} \mathrm{E}$ ). Limité par la zone tellienne au Nord et l'Atlas central au Sud, ce plan d'eau artificiel est situé dans la basse vallée de l'oued Majarda, principal cours d'eau permanent de la Tunisien, qui prend sa source à $1000 \mathrm{~m}$ d'altitude dans la région de Souk Ahras, Algérie. Le climat est de type méditerranéen sub-humide à hiver doux ; les températures moyennes minimales sont atteintes en janvier (entre 5 et $8^{\circ} \mathrm{C}$ ). Le climat est caractérisé par de fortes variations du régime des précipitations réparties de manière inégale sur trois saisons. La moyenne annuelle pour la région de Béja est de $645 \mathrm{~mm} / \mathrm{an}$ entre 1901 et 1960. Certains paramètres physico-chimiques des eaux ont largement fluctué au cours de la période 1989/1997. A titre d'exemple, la teneur moyenne en nitrates est passée de $2.15 \mathrm{mg} / \mathrm{l}$ (1989) à $0.03 \mathrm{mg} \mathrm{N} / \mathrm{l}$ (1997); les eaux ont présenté une salinité maximale en 1989 (1781 $\mathrm{mg} / \mathrm{l})$ et minimale en 1996 (1149 mg/l). En revanche, les teneurs en phosphore total ont été relativement stables $(0.05$ à $0.03 \mathrm{mg} P / 1)$.

\section{Matériel et méthodes}

Le matériel biologique a été récolté lors de nombreuses prospections effectuées de mars 1995 à décembre 1997 en différents points du plan d'eau. Les prélèvements ont été effectués avec un filet de $25 \mathrm{~cm}$ de diamètre d'ouverture (vide de maille : $200 \mu \mathrm{m}$ ). Les échantillons ont été fixés immédiatement au formol (concentration finale : $5 \%$ ). Au laboratoire, les individus sont montés dans la glycérine et observés à l'aide d'un microscope. Après dissection, les appendices sont montés entre lame et lamelle et dessinés à l'aide d'une chambre claire.

Les différentes mesures morphométriques ont été réalisées au microscope muni d'un micromètre oculaire sur un échantillon de 30 femelles prélevé le 25 octobre 1995 par nous-mêmes et 30 femelles provenant d'un échantillon récolté par Sohlobji le 25 septembre 1989 et déposé au Laboratoire d'Aquaculture et Milieu de l'Institut National d'Agronomie de Tunis.

\section{Caractéristiques de Mesocyclops ogun- nus en Tunisie}

Synonymies :

Mesocyclops ogunnus Onabamiro, 1957

Mesocyclops leuckarti (part.) Dussart \& Gras, 1966

Mesocyclops leuckarti (part.) Van de Velde, 1978

Mesocyclops thermocyclopoides (part.) (non Harada) Kiefer, 1981

Mesocyclops sp. 1 Dumont et al., 1981

Mesocyclops ogunnus Van de Velde, 1984.

La longueur du corps des femelles de Mesocyclops ogunnus varie entre 0,95 et $1,22 \mathrm{~mm}$. La membrane hyaline du segment 17 de l'antennule présente une profonde échancrure (Fig. 1A) ; l'ornementation du basipodite de l'antenne est typique de l'espèce (Fig. 1B, 
1C). Le palpe maxillulaire porte une rangée oblique de fortes spinules (Fig. 1D). La maxille présente une rangée de moins de 10 petites spinules sur la face frontale du coxopodite (Fig. 1E).

Le basipodite de $\mathrm{P} 1$ ne porte pas d'épine à l'angle interne.

La lame précoxale de $\mathrm{P} 4$ présente des proéminences dépassant à peine le bord distal. L'article terminal de l'endopodite 3 de P4, 2,5 à 3,3 fois plus long que large, est muni de 2 épines apicales de taille égale, l'interne étant parfois plus courte (Fig. 1F). La partie interne du basipodite de P4 (face caudale) est ornée de deux rangées de fines soies (Fig. 1G).

Les bords latéraux du $5^{\mathrm{e}}$ segment thoracique sont garnis de soies (Fig. 1H). La P5 présente 2 articles dont le distal porte une épine sétiforme latérale en position sub-médiane et une soie terminale 1,1 à 1,5 fois plus longue que l'épine latérale (Fig. 1H).

Le réceptacle séminal est typique et correspond à la description de Van de Velde (1984).

Les branches de la furca, à bords internes glabres, sont 2,4 à 3,1 fois plus longues que larges (Fig. 1I). Une rangée continue de spinules borde la base de chaque branche furcale; quelques spinules à l'insertion de la soie médiane externe (Tme) et de la soie terminale interne (Ti).

Le tableau 1 présente nos valeurs comparées à celles de Onabamiro (1957), Van de Velde (1984), Jeje (1988) et Mirabdullayev (1996). Les légères différences observées portent sur la longueur relative de la soie dorsale de la furca qui est toujours égale ou supérieure à celle de la soie interne, ainsi que sur la taille de l'épine apicale interne de l'endopodite de la P4 qui est inférieure ou égale à celle de l'épine externe. Hormis ces deux critères, la comparaison morphométrique ne révèle pas de différences significatives entre les diverses populations (Tableau 1).
Nous avons observé, dès la fin de l'année 1995, la raréfaction de $M$. ogunnus dans les eaux de la retenue de Sidi Salem. En effet, dans le matériel du 25 septembre 1989, cette espèce est très abondante et Sohlobji (1992 : sub Mesocyclops leuckarti) lui attribue une forte présence au cours de l'année 1989/1990 alors que dès le début de nos investigations (mars 1995) l'espèce était déjà plus rare avec une fréquence d'apparition dans les échantillons de $34 \%$. Au cours des années 1997 et 1998, M. ogunnus n'a été récolté que de manière épisodique ( $-5 \%$ des échantillons) dans la zone pélagique de la retenue. En revanche, Acanthocyclops robustus, très rare en 1989/1990 (Sohlobji 1992), est fréquent depuis 1996 et cohabite avec Thermocyclops oblongatus qui, jusqu'alors, semblait inexistant dans la retenue de Sidi Salem.

\section{Discussion}

Les spécimens tunisiens sont conformes aux descriptions faites par Van de Velde (1984) et particulièrement à l'ornementation du coxopodite de la maxille dessinée par Holynski \& Fiers (1994). Il faut cependant souligner la variabilité plus grande du rapport longueur/largeur des branches furcales qui peut être attribuée aux variations écologiques, phénomène déjà observé chez d'autres espèces. Mesocyclops ogunnus est une espèce dulçaquicole apte à proliférer en milieu oligohalin, saumâtre (Bonou et al. 1991) ou salé (Van de Velde 1984) ; Bonou et al. (1991) constätent ainsi des différences en comparant des populations provenant du lac Tchad et des étangs de Layo (Côte d'Ivoire).

L'identification de Mesocyclops ogunnus par l'un d'entre nous (D.D) dans la retenue de Sidi Salem apporte des informations supplémentaires sur la répartition du genre Mesocyclops et confirme que la Tunisie constitue un point de rencontre de faunes d'origines multiples (éthiopienne, saharo-sindienne, paléarctique) (Mouelhi et al. 1999). La première mention de

Tableau 1. Morphométrie comparative des femelles de Mesocyclops ogunnus d'après quelques auteurs.

Table 1. Measurements of Mesocyclops ogunnus females according to different authors.

\begin{tabular}{|c|c|c|c|c|c|c|}
\hline & \multirow{2}{*}{$\begin{array}{l}\text { Longueur } \\
\text { totale }(\mathrm{mm})\end{array}$} & \multicolumn{2}{|c|}{ Enp3 P4 } & \multirow{2}{*}{$\begin{array}{l}\text { Art. distal P5 } \\
\text { Ep lat/S term }\end{array}$} & \multicolumn{2}{|c|}{ Branches furcales } \\
\hline & & $\mathrm{L} / 1$ & Ep int/Ep ext & & $\mathrm{L} / 1$ & $\mathrm{Te} / \mathrm{Sd}$ \\
\hline Onabamiro (1957) & $1,00-1,30$ & $2,50-3,00$ & 1 or $>1$ & & $3,10-3,30$ & \\
\hline Van de Velde (1984) & 0,989 & 2,46 & 1,04 & & 2,70 & \\
\hline Jeje $(1988)(n=10)$ & $0,90-1,20$ & $2,50-3,20$ & 1 & $0,76-0,80$ & $2,69-3,20$ & $1,35-1,37$ \\
\hline Mirabdullayev (1996) & $1,00-1,15$ & $2,57-3,24$ & & & $2,70-3,13$ & \\
\hline Présente étude $(\mathrm{n}=60)$ & $0,95-1,22$ & $2,50-3,33$ & $0,86-1,0$ & $0,65-0,88$ & $2,42-3,09$ & $0,89-1,11$ \\
\hline
\end{tabular}



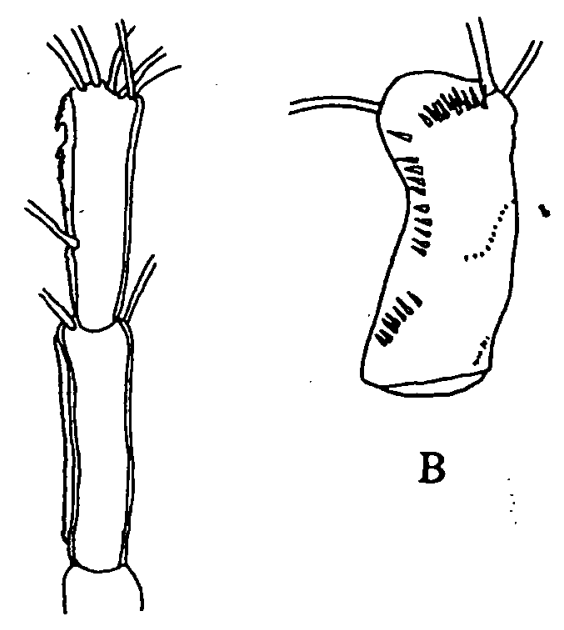

B
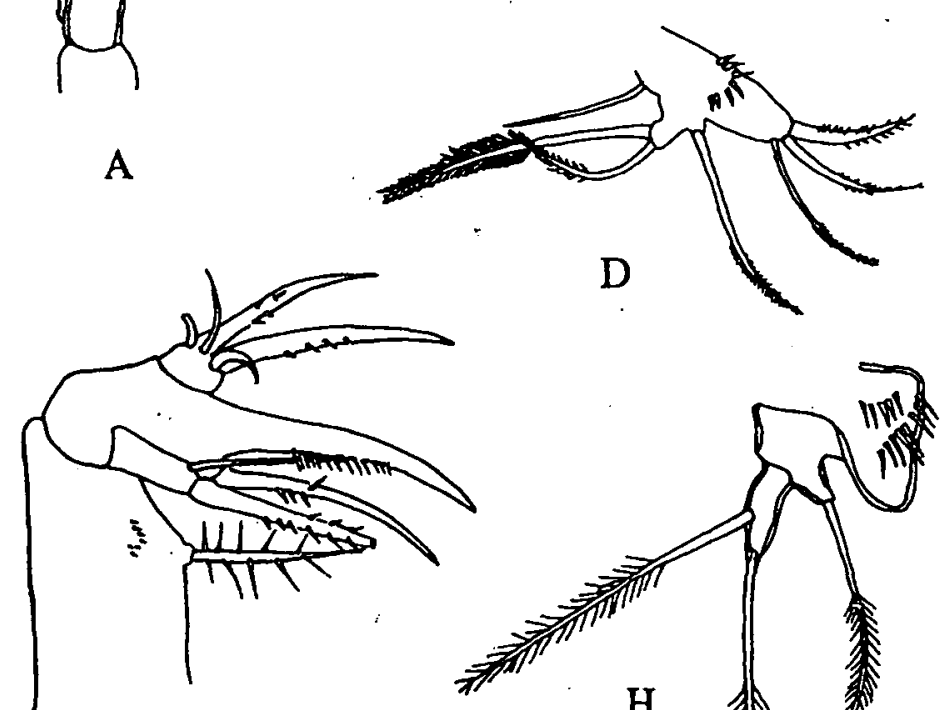

$\mathrm{E}$
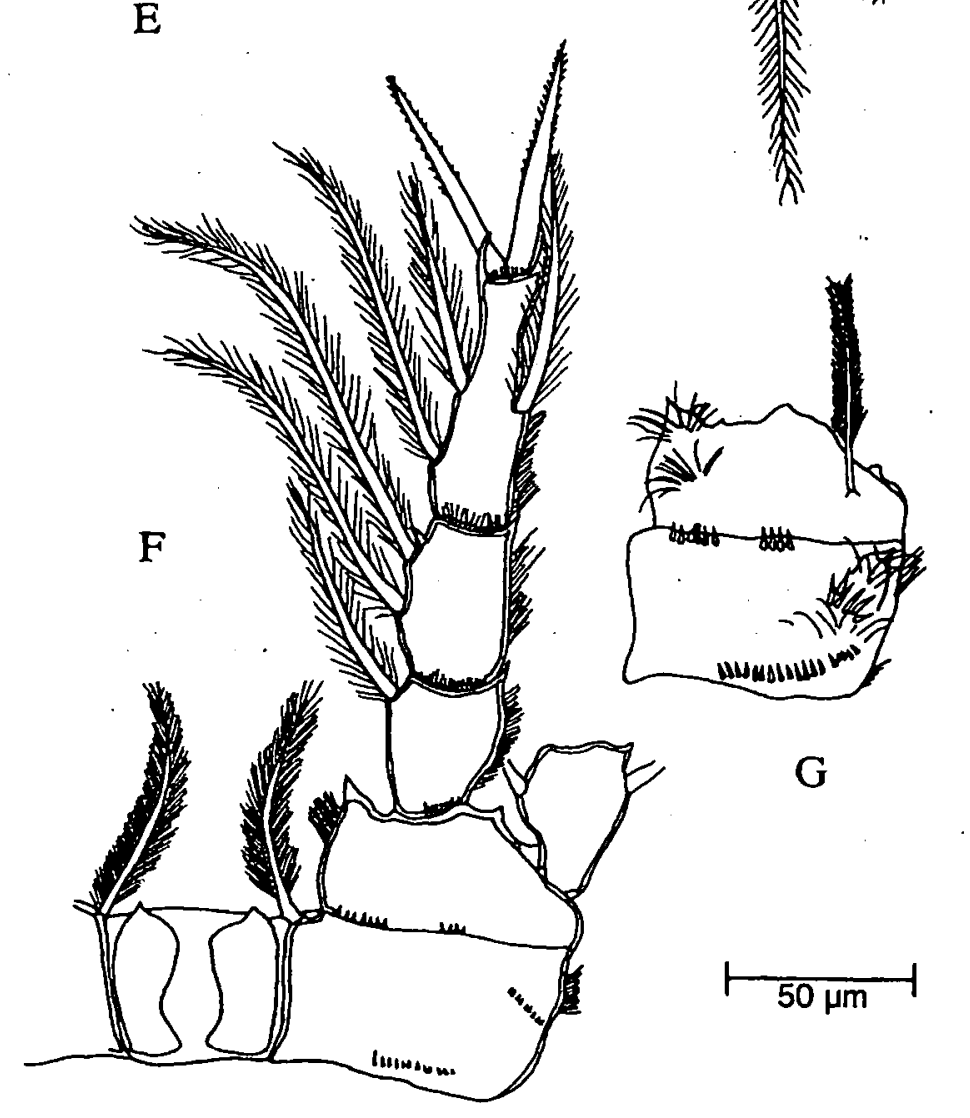

G

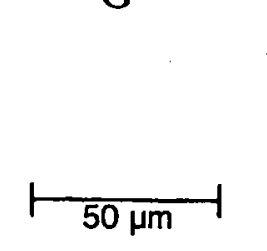

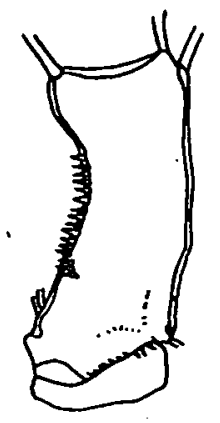

C

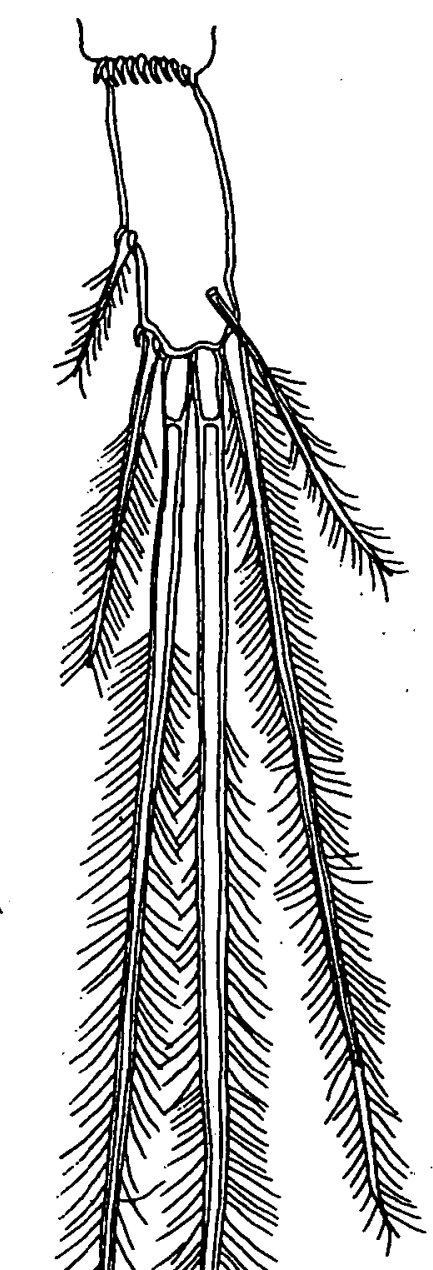

I

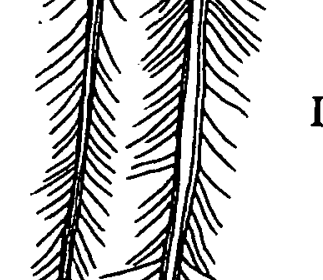


cette espèce dans le Maghreb algérien (Samraoui et al. 1998) puis sa découverte en Tunisie permettent d'établir une jonction entre l'aire de répartition africaine et les observations faites sur le continent euro-asiatique et apportent un argument supplémentaire soulignant les affinités biogéographiques existant entre ces deux domaines.

Cependant, le fait que Mesocyclops n'ait pas été trouvé auparavant peut être sujet à discussion (voir Samraoui et al. 1998). Les populations de ces cyclopoïdes seraient fluctuantes et leur durée de vie limitée, d'où la possibilité d'un échantillonnage à un moment où elles sont absentes du milieu. L'autre hypothèse serait une arrivée très récente de Mesocyclops en Afrique du Nord, c'est-à-dire dans la seconde moitié du $20^{\mathrm{e}}$ siècle (Samraoui et al. 1998). Dans ce dernier cas, il est fort probable que $M$. ogunnus voit son aire de répartition s'étendre au pourtour méditerranéen dans son ensemble.

La raréfaction de $M$. ogunnus dans la retenue de $\mathrm{Si}$ di Salem ne peut pas être raisonnablement expliquée par le changement des conditions abiotiques du milieu et ce, malgré une nette diminution de la salinité et de la teneur en nitrates (Kraïem, comm. pers.). Une compétition interspécifique avec Thermocyclops oblongatus ou Acanthocyclops robustus constitue une hypothèse envisageable bien qu'aucun élément ne la confirme. Cependant, dans le lac Gronne en Allemagne, Maier (1989) observe que $T$. crassus est capable d'éliminer $M$. leuckarti. Si on accepte l'idée que $M$. ogunnus peut coloniser très rapidement un biotope nouveau (espèce pionnière), sa survie dans le milieu dépend peut-être des autres espèces présentes.

\section{Travaux cités}

Bonou C.A., Pagano M. \& Saint-Jean L. 1991. - Développement et croissance en poids de Moina (cf) micrura et de Mesocyclops ogunnus dans un milieu saumâtre tropical : les étangs de pisciculture de Layo (Côte d'Ivoire). Rev. Hydrobiol. trop., 24 (4) : 287-303.

Defaye D. 1988. - Contribution à la connaissance des Crustacés Copépodes d'Ethiopie. Hydrobiologia, 164 : 103-147.

Defaye D. 1991. - Présence de Mesocyclops rarus Kiefer, 1981 (Crustacé, Copépode) au Sénégal. Rev. Hydrobiol. trop., 24 (4) 357-360.

Dumont H.J. \& Maas S. 1988. - Copepods of the lagune Ebrié (Côte d'Ivoire). Rev. Hydrobiol. trop., 21 (1) : 3-7.

Dumont H.J., Pensaert J. \& Van de Velde I. 1981. - The crustacean zooplankton of Mali (West Africa). Hydrobiologia, 80 : 161-187.

Dussart B. 1969. - Les Copépodes des eaux continentales d'Europe occidentale. II. Cyclopoïdes et biologie. Boubée \& Cie (éd.) Paris : $267 \mathrm{p}$.

Dussart B.H. \& Defaye D. 1985 . - Répertoire mondial des Copépodes Cyclopoïdes. CNRS (éd.) Paris : $236 \mathrm{p}$.

Dussart B.H. \& Fernando C.H. 1985. - Les copépodes en Sri Lanka (Calanoïdes et Cyclopoïdes). Hydrobiologia, $127: 229-252$.
Dussart B.H. \& Fernando C.H. 1988. - Sur quelques Mesocyclops (Crustacea, Copepoda). Hydrobiologia, $157:$ 241-264.

Dussart B.H. \& Gras R. 1966. - Faune planctonique du lac Tchad. 1. Crustacés Copépodes. Cah. ORSTOM, série Océanogr., 4 (3) : 77-91.

Gauthier H. 1928. - Recherches sur la faune des eaux continentales d'Algérie et de la Tunisie. Thèse, Minerva, Alger : 419 p.

Gophen M. 1988. - Changes of Copepoda populations in Lake Kinneret during 1969-1985. Hydrobiologia, 167/168 : 375-379.

Holynski M. \& Fiers F. - Mesocyclops thermocyclopoides speciesgroup : redefinition and content. Hydrobiologia, 292/293:41-51.

Jeje C.Y. 1988. - A revision of the Nigerian species of the genera Mesocyclops Sars, 1914 and Thermocylops Kiefer, 1927 (Copepoda : Cyclopoida). Hydrobiologia, 164 : 171-184.

Kiefer F. 1981. - Beitrag zur Kenntnis von Morphologie, Taxomonie und geographischer Verbreitung von Mesocyclops leuckarti auctorum. Arch. Hydrobiol., Suppl. 62 (1) : 148-190.

Maier G. 1989. - The seasonal cycle of Thermocyclops crassus (Fischer, 1853) (Copepoda : Cyclopoida) in a shallow, eutrophic lake. Hydrobiologia, $178:$ 43-58.

Mirabdullayev I.M. 1996. - The genus Mesocyclops (Crustacea : Copepoda) in Uzbekistan (Central Asia). Int. Rev. ges. Hydrobiol., 81 (1) : 93-100.

Mouelhi S., Balvay G. \& Kraïem M.M. 1999. — Le zooplancton des eaux continentales de Tunisie : inventaire préliminaire et biogéographie. Archs Sci. Genève, 52 (2) : 1-12.

Onabamiro S.D. 1952. - Four new species of Cyclops s.l. (Crustacea : Copepoda) from Nigeria. Proc. zool. Soc. Lond., 122 (1) : 253-266.

Onabamiro S.D. 1957. - Some new species of Cyclops sensu lat. (Crustacea : Copepoda) from Nigeria. J. linn. Soc. Lond., 43 : 123-133.

Pagano M. \& Saint-Jean L. 1994. - Le zooplancton. In : J.R. Durand, P. Dufour, D. Guiral \& S.G.F. Zabi (eds), Environnement et ressources aquatiques de Côte-d'Ivoire. II. Les milieux lagunaires, Editions ORSTOM Paris : 155-188.

Por F.D. 1984. - An outline of the distribution patterns of the freshwater Copepoda of Israel and surroundings. Hydrobiologia, $113: 151-154$.

Reid J.W. \& Kay B.H. 1992. - Mesocyclops guanxiensis, new species, and new records of four congeners (Crustacea : Copepoda : Cyclopoida) from China, Laos, and Viet Nam. Proceed. Biol. Soc. Washington, $105: 331-342$.

Reid J.W. \& Pintho-Coelho R.M. 1994. - An Afro-Asian continental copepod, Mesocyclops ogunnus, found in Brazil, with a new key to the species of Mesocyclops in south America and a review of intercontinental introductions of copepods. Limnologica, $28: 359-368$.

Roy J. \& Gauthier H. 1927. - Sur les copépodes d'Algérie et de Tunisie (eaux douces et saumâtres). Bull. Soc. zool. Fr., 52 : 558-575.

Samraoui B., Segers H., Maas S., Baribwegure D. \& Dumont H.J., 1998. - Rotifera, Cladocera Copepoda, and Ostracoda from coastal wetlands in northeast Algeria. Hydrobiologia, 386 : 183-193.

Sohlobji D. 1992. — Le barrage de Sidi Salem. Conditions du milieu et études zooplanctoniques. DEA, Univ. Tunis II : $183 \mathrm{p}$.

Sohlobji D., Zaouali J., Ben Rejeb-Jenhani A. \& Kartas F. 1993. Microfaune et microflore des eaux du barrage de Sidi Salem (Nord de la Tunisie). Bull. Soc. Sci. nat. Tunisie, 22 : 53-61.

Van de Velde I. 1978. - Cladocera and Copepoda from the Valley of the river Senegal. Biol. Jahrb. Dodonaea, $46: 192-201$.

Van de Velde I. 1984. - Revision of the African species of the genus Mesocyclops Sars, 1914 (Copepoda : Cyclopidae). Hydrobiologia, 109 : 3-66. 